Rev. SINAPSIS, Vol. 5, No 2, Diciembre 2014

ISSN $1390-7832$

\title{
Aprendizaje colaborativo mediante el uso de las wiki en los estudiantes de educación básica
}

\section{Aprendizaje colaborativo en la educación básica}

Rosa Laura Zambrano Molina. Mg Sc. ${ }^{(1)}$

David Segundo Palacios Conforme. Mg Sc. ${ }^{(2)}$

(1) Unidad Educativa Fiscal Portoviejo, Ecuador

(2) Unidad Educativa José Peralta, Portoviejo, Ecuador

Contacto: rosazambranomoli@gmail.com

Receptado: 16/07/2014 Aceptado: 04/09/2014

\section{Resumen:}

El artículo aborda la necesidad del trabajo colaborativo en los estudiantes de educación básica en Ecuador mediante el uso de las Wiki. Para sustentar esta propuesta se realizaron encuestas a docentes y estudiantes de la Unidad Educativa Tiburcio Macías. A través de los resultados obtenidos, se concluyó que existe carencia en el uso de dicha herramienta, se corroboró también que hay una podre aplicación de estrategias para el logro de aprendizaje colaborativo en los estudiantes de educación básica, aspecto que afecta el desarrollo de destrezas y habilidades en los estudiantes, por lo que es necesario llevar a la práctica la estrategia didáctica de aplicación que se propone.

Palabras clave: Comunicación, estrategia didáctica, herramientas tecnológicas trabajo colaboración

\section{Collaborative learning using the wiki in basic education student}

\begin{abstract}
The article discusses the need for collaborative work among students in basic education in Ecuador, using the Wiki. To support this proposal surveys to teachers and students of the School Tiburcio Macias. Through the results, it was concluded that there is a lack in the use of this tool, it was also confirmed that there is pus implementing strategies to achieve collaborative learning among students in basic education, something that affects the
\end{abstract}


Rev. SINAPSIS, Vol. 5, N 2, Diciembre 2014

ISSN $1390-7832$

development of skills and skills in students, so it is necessary to implement the teaching strategy proposed application

Keywords: Communication, teaching strategy, technological collaborative work tools

\section{Introducción}

El aprendizaje colaborativo, de acuerdo a Johnson y Johnson (1999) es un sistema de interacciones cuidadosamente diseñado que organiza e induce la influencia recíproca entre los integrantes de un equipo. Se desarrolla a través de un proceso gradual en el que todos y cada miembro se siente mutuamente comprometido con el aprendizaje de los demás generando una interdependencia positiva que no implique competencia. Se adquiere a través del empleo de métodos de trabajo grupal caracterizado por la interacción y el aporte de todos en la construcción del conocimiento.

En el aprendizaje colaborativo el trabajo grupal apunta a compartir la autoridad, a aceptar la responsabilidad y el punto de vista del otro, a construir consenso con los demás. Para trabajar en colaboración es necesario compartir experiencias, conocimientos y tener una meta grupal por lo que la retroalimentación es esencial para el éxito "Lo que debe ser aprendido sólo puede conseguirse si el trabajo del grupo es realizado en colaboración. Es el grupo el que decide cómo realizar la tarea, qué procedimientos adoptar, cómo dividir el trabajo, las tareas a realizar" (Johnson y Johnson, 1999,)

La implementación de procesos colaborativos conlleva a aplicar todo un procedimiento, ya que intervenir o participar no sólo significa estar en un grupo o conectados virtualmente, sino la aplicación de tiempos, expresar experiencias, criterios, análisis, reflexiones y sacar conclusiones; es decir demanda todo un proceso científico, que puede ser mucho más eficiente con la utilización de los instrumentos de la Web.

Por otra parte, el uso de wikis en educación no está extendido si lo comparamos con otro tipo de aplicaciones y entornos diseñados desde el discurso didáctico dominante. Los wikis pueden ser vistos como una tecnología "rupturista" frente a la estructura unidireccional de la mayoría de las aplicaciones web ya que otorga derechos simétricos a todos los 
Rev. SINAPSIS, Vol. 5, N 2, Diciembre 2014

ISSN $1390-7832$

participantes que basan su éxito en la actividad colectiva de reflexión y comunicación y en la auto-organización de la comunidad (Alonso y Gallego, 1996). Pese a ello, los wikis cada día juegan un papel más destacado dentro de entornos tradicionales. Así diversos sistemas los incorporan como herramienta para la realización de actividades didácticas que requieren algunas de sus características técnicas. A pesar de esto, muchas instituciones educativas poseen laboratorio de computación, internet y tecnología informática, pero se lo utilizan en el aprendizaje de los estudiantes en forma tradicional; las herramientas como la wiki no son utilizadas, limitando el desarrollo de habilidades para buscar, obtener, procesar y comunicar información, y posteriormente transformarla en conocimiento.

Los estudiantes, sobre todo los de educación básica son muy susceptibles de lo nuevo, de manejar las redes sociales con mucha mayor eficacia que los docentes, por ello, incorporar la wiki a su educación genera efectos positivos, que sin lugar a dudas se están desperdiciando en este nivel por considerarse muchas veces que los niños no son capaces de saber utilizar estas herramientas.

Teniendo en cuenta estas premisas, el trabajo se encuentra basado hacia el objetivo de proponer estrategias didácticas para desarrollar las clases utilizando las wikis como herramienta del aprendizaje colaborativo.

\section{Método}

Para el desarrollo del trabajo, se utilizó el apoyo bibliográfico para la fundamentación teórica del mismo, donde se consultaron teorías relacionadas al tema. Se realizó una investigación de campo. Esta consistió en la aplicación de encuestas a estudiantes y docentes de la Unidad Educativa Tiburcio Macías de la ciudad de Portoviejo, Ecuador.

\section{Resultados y discusión}

De la triangulación de los principales resultados alcanzados por cada uno de los métodos e instrumentos utilizados, se llega a las consideraciones que se expresan a continuación: 
Rev. SINAPSIS, Vol. 5, N 2, Diciembre 2014

ISSN $1390-7832$

En relación a la utilización de la wikis como medio de comunicación, un alto porcentaje de docentes encuestados manifiestan que no lo han utilizado ni de manera personal, menos como herramienta educativa. De la misma manera los estudiantes de forma general corroboran que en su proceso de aprendizaje no utilizan estas herramientas, ni siquiera la conocen.

De acuerdo a esto, Bruns y Humphreys (2005) proponen el uso de wikis en educación como espacios de comunicación para desarrollar algunas de las habilidades y, sobre todo actitudes de un nuevo tipo de alfabetización tecnológica que denominan "crítica, colaborativa y creativa" y que va más allá del mero dominio instrumental de las herramientas y entornos de comunicación que ofrecen las nuevas tecnologías de la información y la comunicación. Existen interconexiones y sinergias entre el contexto amplio de la nueva economía y sus estructuras de red y el movimiento hacia modelos pedagógicos socio-constructivistas en educación que emplean estrategias de aprendizaje sociales y colaborativas basadas en proyectos" (Bruns y Humphreys, 2004).

De acuerdo a los resultados, un considerable grupo de maestros considera que los sistemas informáticos de comunicación permiten un aprendizaje colectivo; de este mismo grupo hay quienes coinciden que el aprendizaje que se produce es el individualizado y el colaborativo. De la misma manera la mayoría de los alumnos encuestados expresan que la wiki le ayudaría a educarse en grupo, así como intercambiar información con los compañeros de clases.

Crook (1998) expresa que el aprendizaje se genera a partir de la combinación de una serie de principios: la articulación, el conflicto y la co-construcción para lo cual el docente tiene que diseñar cuidadosamente la propuesta, definir objetivos, los materiales de trabajo, dividir el tópico a tratar en sub tareas, oficiar de mediador cognitivo en cuanto a proponer preguntas esenciales y subsidiarias que realmente apunten a la construcción del conocimiento y no a la repetición de información obtenida y, finalmente monitorear el trabajo resolviendo cuestiones puntuales individuales o grupales según el objetivo de la clase. 
Rev. SINAPSIS, Vol. 5, No 2, Diciembre 2014

ISSN $1390-7832$

Como pedagogía, el trabajo colaborativo comprende el espectro entero de las actividades de los grupos de estudiantes, que trabajan juntos en clase y fuera de ella. Como método puede ser muy formalmente estructurado, como en el proceso que actualmente conocemos como aprendizaje cooperativo o simple e informal como cuando los estudiantes discuten sus ideas entre ellos buscando alguna respuesta consensual, para después compartirla con sus colegas. Este conjunto de métodos de instrucción y de entrenamiento se apoyan en la tecnología y en estrategias que permiten desarrollar en el alumno habilidades personales y

sociales logrando que cada integrante del grupo se sienta responsable no sólo de su aprendizaje, sino del de los restantes miembros del grupo. (Lucero, Margarita 2003).

\section{PROPUESTA DE APLICACIÓN DE ESTRATEGIAS DIDACTICAS EN EL AULA}

A continuación se expone una serie de estrategias a desarrollar en el aula cuando se trabaja con wikis

\section{Antes de iniciar la unidad didáctica, actividad o tarea}

- Partir de una actividad de motivación o un tema motivador para el alumno.

- Procurar que el alumno pueda atribuir sentido a todo lo que le proponemos aprender.

- Explicar bien los objetivos que pretendemos conseguir.

- Comprobar que el alumno entiende lo que se le pide.

- Movilizar los conocimientos previos que el alumno tiene sobre el objeto de aprendizaje.

\section{Durante la unidad didáctica, actividad o tarea}

- Fomentar actitudes y ofrecer estrategias para que el alumno pueda ser cada vez más autónomo al procesar la información.

- Ayudar al alumno para que sea capaz de compartir la construcción del conocimiento y comunicar la información.

- Ofrecer estrategias de cooperación (el docente puede ser un buen modelo para esto).

- Ofrecer estrategias de autoevaluación y coevaluación.

\section{Al finalizar la unidad didáctica, actividad o tarea}

- Recapitular lo aprendido. 
Rev. SINAPSIS, Vol. 5, N 2, Diciembre 2014

ISSN $1390-7832$

- Recuperar o ampliar.

- Presentar el trabajo final realizado.

- Valorar el trabajo realizado.

\section{Conclusiones}

Según el análisis efectuado de la necesidad del trabajo colaborativo en los estudiantes de educación básica mediante el uso de las Wiki, se concluyó que tanto los docentes como los estudiantes no utilizan esta herramienta y que existe una desvinculación en el desarrollo de destrezas y habilidades relacionadas con el uso de la tecnología. Es necesario que se fomente la práctica del trabajo colaborativo a través de un proceso formativo permanente en el aula de clases para que exista un fortalecimiento en la educación integral de los niños y jóvenes.

\section{Bibliografía}

1. Alonso, C. y Gallego, D. (1996) Formación del profesor en Tecnología Educativa, en Gallego, D. y otros: Integración curricular de los recursos tecnológicos. Barcelona, Oikos-Tau, p. 31-64.

2. Bruns, A. y Humphreys, S. (2005). Wikis in Teaching and Assessment: The M/Cyclopedia.

3. Brunner, J.J. (2003). Educación e Internet. ¿La próxima revolución?- Santiago de Chile, Fondo de Cultura Económica.

4. Crook, Ch. (1998). Ordenadores y aprendizaje colaborativo. España. Ediciones Morata.

5. Gómez, M y Pozo, J (1998) Aprender en enseñar ciencia. Madrid: Ediciones Morata.

6. Johnson, D. y R. Johnson (1989) Cooperación y Competencia.

7. Lucero M, et al, (2003). Modelo de Aprendizaje Colaborativo en el Ambiente.

8. Moreira, A. (2005) Otra forma de enseñar. Universidad de México. Edit. UNM. I Edición. 
Rev. SINAPSIS, Vol. 5, No 2, Diciembre 2014

ISSN $1390-7832$

Solomon, C. (1987).Entornos de aprendizaje con ordenadores. Una reflexión sobre las teorías del aprendizaje y la educación. Barcelona: Paidós/MEC. 